\title{
ORIGINAL ARTICLE Effect of rugby training on blood antioxidant defenses in able-bodied and spinal cord injured players
}

\author{
E Hübner-Woźniak ${ }^{1}$, N Morgulec-Adamowicz ${ }^{2}$, M Malara $^{1}$, P Lewandowski $^{1}$ and J Okęcka-Szymańska ${ }^{1}$
}

Study design: A cross-sectional study with comparison group.

Objectives: To examine the effect of rugby training on the blood antioxidant capacity in able-bodied and wheelchair rugby players with tetraplegia.

Setting: Poland.

Methods: Four groups of subjects participated in the study: sedentary able-bodied males (group SA, $n=19$ ), sedentary males with tetraplegia (group ST, $n=10$ ), able-bodied rugby players (group RA, $n=22$ ) and wheelchair rugby players with tetraplegia (group RT, $n=14$ ). The activities of superoxide dismutase (SOD), glutathione reductase (GR) and catalase (CAT) were determined in erythrocyte hemolysates, whereas glutathione peroxidase (GPX) activity was determined in whole-blood hemolysates. Concentrations of total antioxidant status (TAS) was determined in plasma.

Results: SOD activity was significantly higher in the group SA compared with group ST and group RA. No significant differences occurred within the tetraplegic groups: RT and ST. Resting CAT and GPX activities were significantly higher in both the groups of rugby players than in the respective group of sedentary males. There were no differences in GR activity among all the studied groups. Plasma TAS concentration was higher in both the groups of able-bodied males compared with the respective groups of tetraplegics. The present study is the first to conduct an evaluation of wheelchair rugby training-induced adaptations to oxidative stress in individuals with tetraplegia.

Conclusion: Adaptive response to training was similar in both able-bodied and wheelchair rugby players, and it was characterized by increased erythrocyte CAT and GPX activities in resting conditions improving resistance to oxidative stress.

Spinal Cord (2012) 50, 253-256; doi:10.1038/sc.2011.134; published online 29 November 2011

Keywords: antioxidant enzymes; antioxidants in plasma; sedentary men; rugby players; wheelchair rugby players; tetraplegia

\section{INTRODUCTION}

Wheelchair rugby is becoming increasingly popular among individuals with tetraplegia. It can be adapted to a wide range of individual abilities, and combines short intense sessions of exercise over an extended playing time, therefore it can improve aerobic as well as anaerobic capabilities. ${ }^{1}$ Rugby is a sport involving frequent bouts of high-intensity exercise interlaced with low-intensity exercise. The physiological demands of rugby are complex, requiring players to develop speed, muscular strength and power, as well as high aerobic power. ${ }^{2}$ Thus, it is a sport in which both aerobic and anaerobic energy systems are utilized.

It is well known that regular physical exercise affects antioxidantpro-oxidant balance possibly by reducing reactive oxygen species (ROS) and/or increasing antioxidant activity levels. Exercise-induced adaptations may signal cell protection and possibly decrease the occurrence of diseases associated with oxidative stress. ${ }^{3}$ Most of the studies have implicated aerobic exercise as a major cause of increased oxidative stress, but a growing body of evidence shows that highintensity anaerobic exercise can also cause increased production of ROS. ${ }^{4}$ It has been suggested that, specifically to anaerobic exercise, oxidative stress is mediated not only through electron leakage from mitochondrial electron-transport chain but also through other pathways, such as xanthine and NADPH oxidase production, phagocytic respiratory burst activity and disruption of iron-containing proteins. ${ }^{5}$ There is much evidence that ROS production during exercise can initiate health promoting cell signalling and adaptive processes that results in increased activity of antioxidant enzymes. ${ }^{6}$

ROS production in cells is physiologically balanced with the activities of antioxidant systems. Antioxidative defence of the organism includes enzymatic and non-enzymatic systems consisting of antioxidant enzymes, such as superoxide dismutase (SOD; E.C.1.15.1.1.), catalase (CAT; E.C.1.11.1.6.), glutathione peroxidase (GPX; E.C.1.11.1.9.) and glutathione reductase (GR; E.C.1.6.4.2.), as well as the low-molecular endogenous antioxidants, such as reduced glutathione, uric acid and exogenous antioxidants, mainly $\beta$-carotene, retinol, vitamin $\mathrm{C}$ and $\alpha$-tocopherol. ${ }^{7}$ ROS has an important physiological function in organisms by participating in cellular signalling pathways, initiating cellular senescence and apoptosis, stimulating of antioxidant and repair processes. ${ }^{3}$ However, excessive ROS may be involved in a number of diseases, such as atherosclerosis, rheumatic arthritis and cancer, as well as ageing. ${ }^{8}$

Spinal cord injury (SCI) impairs the ability of individuals with tetraplegia to perform large muscle mass aerobic exercise. This is

${ }^{1}$ Department of Biochemistry, Józef Piłsudski University of Physical Education in Warsaw, Warsaw, Poland and ${ }^{2}$ Department of Adapted Physical Activity, Józef Piłsudski University of Physical Education in Warsaw, Warsaw, Poland

Correspondence: Dr N Morgulec-Adamowicz, Department of Adapted Physical Activity, Józef Piłsudski University of Physical Education in Warsaw, ul. Marymoncka 34, 00-968 Warsaw, Poland.

E-mail: natalia.morgulec@awf.edu.pl

Received 7 June 2011; revised 26 September 2011; accepted 1 October 2011; published online 29 November 2011 
caused by the impairment of the function of arms, legs and pelvic organs. The degree of impairment is determined by the neurological level of SCI, however, the physiological and metabolic responses to exercise of athletes with SCI are similar to those for able-bodied athletes but at lower absolute levels. ${ }^{9}$ The disturbances in blood flow in individuals with tetraplegia result in ischemia-reperfusion injury associated with increased production of radical oxygen species. ${ }^{3}$ It is well known that regular physical activity increases antioxidant capacity as a result of adaptation changes of antioxidant enzyme activities and quite likely a lower ROS during submaximal exercise. ${ }^{3}$ Barfield et al. ${ }^{10}$ recommend wheelchair rugby as a viable approach to improving cardio-respiratory fitness. There are no data concerning the adaptive effect of blood antioxidant enzymes activity in wheelchair rugby players. Therefore, the aim of the present study was to examine the effect of rugby training on the erythrocyte antioxidant enzymes activity and on antioxidants in plasma in able-bodied and wheelchair rugby players with tetraplegia.

\section{MATERIALS AND METHODS}

\section{Subjects}

Four groups of subjects participated in the study: sedentary able-bodied males (group SA, $n=19$ ), sedentary males with tetraplegia (group ST, $n=10$ ), semiprofessional able-bodied rugby players (group RA, $n=22$ ) and wheelchair rugby players with tetraplegia (group RT, $n=14$ ). A personal questionnaire was completed to obtain the following data: age, height, neurologic motor level, the American Spinal Cord Injury Association (ASIA) impairment scale and training experience. A total of 16 individuals with tetraplegia were classified as having an ASIA A injuries ( 9 in group ST and 7 in group RT) and 8 ASIA B injuries ( 4 in group ST and 4 in group RT). The neurological motor level in the group ST was C5 in two individuals (20\%), C6 in five individuals (50\%) and C7 in three individuals (30\%). In the group RT the neurological motor level was C5 in three individuals (22\%), C6 in seven individuals $(50 \%)$ and C7 in four individuals (28\%). Group RA had training sessions five times a week ( $2-3 \mathrm{~h}$ per session), whereas the training schedule for group RT was two sessions a week (2$3 \mathrm{~h}$ per session). Rugby players refrained from physical exercise for at least $48 \mathrm{~h}$ before blood collection. Physical characteristics of all groups are shown in Table 1. All subjects gave their written consents to participate in the study, which was approved by the local Ethics Committee of the Józef Piłsudski University of Physical Education in Warsaw, according to the Declaration of Helsinki.

\section{Instrumentation}

Body mass was measured with use of scale weight type WPT 8.300BC (Radwag, Radom, Poland). Body fat was determined by single-site NIR (Futrex-6100 A/ZL, Futrex Inc., Gaithersburg, MD, USA) as described previously. ${ }^{11}$ Each subject sat with his dominant arm relaxed on an examination table, whereas the light wand of the NIR was placed on the biceps muscle at the mid point between the elbow and the shoulder. Then, the body mass index (BMI=body mass in $\mathrm{kg} / \mathrm{height}$ in $\mathrm{m}^{2}$ ) was calculated.

Blood samples were collected in the preprandial state from the antecubital vein into heparinized tubes. Aliquot of the collected blood was centrifuged at $3000 \mathrm{~g}$ for $15 \mathrm{~min}$ at $4{ }^{\circ} \mathrm{C}$ to separate plasma, and the erythrocytes were washed three times with cold saline. Whole blood, erythrocytes and plasma were frozen at $-70^{\circ} \mathrm{C}$ until assayed.

The activity of SOD was determined in erythrocyte hemolysates incubated with xanthine and xanthine oxidase to generate superoxide radicals, which react with 2-(4-iodophenyl)-5-phenyltetrasodium chloride to form red formazon dye by using commercially available kit (Ransod; Randox, Crumlin, UK). The interassay variability was $4.8 \%$. GPX was determined in whole-blood hemolysates using commercial kit (Ransel; Randox). This assay used an enzyme-coupled reaction and measured the oxidation of NADPH by cumene hydroperoxide as substrate. The inter-assay variability was $4.3 \%$. The activity of GR was assayed in erythrocyte hemolysate incubated with glutathione (GSSG) in the presence of $\mathrm{NADPH}$, which is oxidized to $\mathrm{NADP}^{+}$(commercial kit, Randox). The interassay variability was $3.8 \%$. The activity of CAT was measured in erythrocyte hemolysates using commercially available kit (Oxis, Foster City, CA, USA).
Table 1 Physical characteristics of studied groups (mean \pm s.d.)

\begin{tabular}{lccccc}
\hline \multirow{2}{*}{ Variable } & \multicolumn{2}{c}{ Sedentary } & & \multicolumn{2}{c}{ Rugby players } \\
\cline { 2 - 3 } \cline { 5 - 6 } & $S A(\mathrm{n}=19)$ & $S T(\mathrm{n}=10)$ & & $R A(\mathrm{n}=22)$ & $R T(\mathrm{n}=14)$ \\
\hline Age (years) & $30.0 \pm 4.2$ & $26.9 \pm 5.2$ & & $23.0 \pm 5.0^{\mathrm{a}}$ & $30.5 \pm 5.4$ \\
Body height $(\mathrm{cm})$ & $179.4 \pm 7.5$ & $179.9 \pm 6.5$ & & $181.0 \pm 5.9$ & $184.9 \pm 11.0^{\mathrm{b}}$ \\
Body mass (kg) & $83.7 \pm 14.4$ & $74.9 \pm 12.2$ & & $90.4 \pm 13.7$ & $77.9 \pm 15.9^{\mathrm{c}}$ \\
BMI & $25.9 \pm 3.0^{\mathrm{d}}$ & $23.1 \pm 3.3$ & & $27.6 \pm 4.3^{\mathrm{e}}$ & $22.9 \pm 3.7$ \\
Fat content (\%) & $19.6 \pm 5.6$ & $16.9 \pm 6.0$ & & $22.5 \pm 5.5^{\mathrm{e}}$ & $15.7 \pm 4.7$ \\
Training & - & - & & $7.6 \pm 3.6$ & $7.1 \pm 3.4$
\end{tabular}

experience (years)

Abbreviations: BMI, body mass index; RA, able-bodied rugby players; RT, wheelchair rugby players with tetraplegia; SA, sedentary able-bodied males; ST, sedentary males with tetraplegia. aSignificantly lower compared with group SA and group RT $(P<0.001)$.

bSignificantly higher compared with group ST and group RA $(P<0.05)$.

cSignificantly lower compared with group RA $(P<0.01)$.

dSignificantly higher compared with group ST $(P<0.01)$.

esignificantly higher compared with group RT $(P<0.01)$.

Samples were incubated with $\mathrm{H}_{2} \mathrm{O}_{2}$, peroxidase and chromogen, which originated from quinoneimine dye. CAT activity was read from the standard curve prepared for samples with known activities of this enzyme. For CAT intra-assay coefficient of variation was $5.6 \%$. Plasma total antioxidant status (TAS) was measured using ABTS (2,2'-anizo-di-[3-ethylbenzthiazoline sulphonate]) incubated with peroxidase and $\mathrm{H}_{2} \mathrm{O}_{2}$ to produce the radical cation $\mathrm{ABTS}^{+}$ (commercial kit, Randox). Antioxidants in the added sample caused suppression of this coloured product to a degree, which is proportional to their concentration. The inter-assay variability was $3.1 \%$. Hemoglobin concentrations in both whole blood and in erythrocyte hemolysates were determined using Drabkin's method. Enzyme activities were expressed in $\mathrm{Umg}^{-1} \mathrm{Hb}$ or $\mathrm{Ug}^{-1} \mathrm{Hb}$.

\section{Data analysis}

Data were reported as mean values and standard deviations. Shapiro-Wilk's test was used to check the normality of distribution in the data analysis. The Student's $t$-test for independent data and the Mann-Whitney's $U$-test were used in cases of normal and non-normal distributions, respectively. The level of significance was set at $P<0.05$.

\section{RESULTS}

Able-bodied rugby players were significantly younger compared with sedentary able-bodied and both sedentary tetraplegics and wheelchair rugby players with tetraplegia (Table 1). The reason for this difference was that the non-disabled rugby players were selected to ensure similar rugby training experience. Body height was significantly higher and body mass and percent of fat content were lower in wheelchair rugby players with tetraplegia (group RT) compared with able-bodied rugby players (group RA). Both sedentary tetraplegics and wheelchair rugby players with tetraplegia were characterized by lower BMI values than the respective able-bodied groups.

In sedentary able-bodied males, SOD activity was significantly higher compared with sedentary males with tetraplegia and ablebodied rugby players; however, in wheelchair rugby players with tetraplegia compared with sedentary individuals with tetraplegia SOD activity was similar (Table 2). Activities of CAT and GPX were significantly higher in both groups of rugby players (47.6\% and $16.9 \%$, respectively, in RA; $28 \%$ and $19.8 \%$, respectively, in RT) than in the corresponding sedentary groups of males. There were no differences in GR activity between groups of able-bodied and tetraplegics. Plasma TAS concentration was higher in both groups of able-bodied males compared with the respective groups of individuals with tetraplegia.

\section{DISCUSSION}

It is well documented that regular physical training is associated with adaptive increase of antioxidant enzyme activities and enhanced 
Table 2 Blood antioxidant enzyme activities and plasma TAS concentration in sedentary males and rugby players, each split into able-bodied and tetraplegics (mean \pm s.d.)

\begin{tabular}{|c|c|c|c|c|}
\hline \multirow[t]{2}{*}{ Variable } & \multicolumn{2}{|c|}{ Sedentary } & \multicolumn{2}{|c|}{ Rugby players } \\
\hline & $S A(\mathrm{n}=19)$ & $S T(\mathrm{n}=10)$ & $R A(\mathrm{n}=22)$ & $R T(\mathrm{n}=14)$ \\
\hline $\operatorname{SOD}\left(\mathrm{Ug}^{-1} \mathrm{Hb}\right)$ & $1374 \pm 509^{a}$ & $1014 \pm 144$ & $1103 \pm 177$ & $990 \pm 194$ \\
\hline CAT (U mg $\left.{ }^{-1} \mathrm{Hb}\right)$ & $214.7 \pm 51.2^{b}$ & $244.3 \pm 58.4^{b}$ & $316.9 \pm 34.3$ & $312.9 \pm 32.6$ \\
\hline $\mathrm{GPX}\left(\mathrm{Ug}^{-1} \mathrm{Hb}\right)$ & $49.7 \pm 12.9^{b}$ & $51.4 \pm 12.4^{b}$ & $58.1 \pm 10.7$ & $61.6 \pm 10.7$ \\
\hline $\mathrm{GR}\left(\mathrm{Ug}^{-1} \mathrm{Hb}\right)$ & $10.2 \pm 1.7$ & $10.4 \pm 1.3$ & $11.3 \pm 2.1$ & $9.9 \pm 2.1$ \\
\hline TAS (mmol $\left.\mathrm{I}^{-1}\right)$ & $1.49 \pm 0.18$ & $1.08 \pm 0.18^{c}$ & $1.49 \pm 0.10^{d}$ & $1.30 \pm 0.19$ \\
\hline
\end{tabular}

Abbreviations: CAT, catalase; GPX, glutathione peroxidise; GR, glutathione reductase; RA, ablebodied rugby players; RT, wheelchair rugby players with tetraplegia; SA, sedentary able-bodied males; SOD, superoxide dismutase; ST, sedentary males with tetraplegia; TAS, total antioxidant status.

aSignificantly higher compared with group ST and group RA $(P<0.05)$

bsignificantly lower compared with the respective groups of rugby players $(P<0.001)$.

c $S$ ignificantly lower compared with group SA $(P<0.001)$ and group RT $(P<0.01)$.

cSignificantly lower compared with group SA $(P<0.001)$ and
dSignificantly higher compared with group RT $(P<0.001)$.

resistance against oxidative stress ${ }^{6,7}$ and lower production of ROS during submaximal exercise. Increased antioxidant enzyme activities were observed as an effect of both aerobic and anaerobic training. ${ }^{12}$ In addition, it is also accepted that interval training, characteristic to team sports, has a similar effect, although the results of studies are often inconsistent. Teixeira et al. ${ }^{13}$ demonstrated that erythrocyte SOD activity was higher in kayakers and canoeists, although no statistically significant differences were observed for GPX and GR activities between athletes and sedentary controls. Also Melikoglu et al. ${ }^{14}$ discovered that SOD and GPX activities were higher in basketball players than in sedentary men. Finaud et al. ${ }^{15}$ stressed that oxidative stress and antioxidant capacity can be modified by magnitude of training loads in team sport such as rugby. Evelson et al. ${ }^{16}$ determined that resting SOD activity in well-trained rugby players was higher compared with sedentary men. In the present study, SOD activity was alike in the groups of sedentary individuals with tetraplegia and wheelchair rugby players with tetraplegia but was lower (approximately $20 \%$ ) in the group of able-bodied rugby players compared with sedentary persons. The lower resting SOD activity in rugby players was somewhat unexpected and had been attributed to a modification of the catalytic site and subsequent inactivation of this enzyme ${ }^{17}$ or decreased erythrocyte SOD activity, even 19 days after finishing longlasting exercise. ${ }^{18}$ It cannot be excluded that SOD activity was lower in able-bodied rugby players due to insufficient intake of zinc and cooper, which are coenzymes of SOD.

GPX activity has been associated with the maximal oxygen uptake attained by specific training, and its increased activity reflects adaptation to the exercise-induced oxidative stress. ${ }^{19}$ Activity of GPX was significantly higher in able-bodied and wheelchair rugby players than in respective groups of sedentary persons. These results indicate a similar response to rugby training in both able-bodied and individuals with tetraplegia. Therefore, it can be suggested that rugby training and wheelchair rugby training can raise athlete endurance capacity. In the present study, it was shown that erythrocyte CAT activities were higher in both the groups of rugby players $\left(316.9 \mathrm{Umg}^{-1} \mathrm{Hb}\right.$ in RA and $312.9 \mathrm{U} \mathrm{mg}^{-1} \mathrm{Hb}$ in RT) compared with respective sedentary males (214.7 $\mathrm{U} \mathrm{mg}^{-1} \mathrm{Hb}$ in SA and $244.3 \mathrm{U} \mathrm{mg}^{-1} \mathrm{Hb}$ in ST).

Plasma TAS level reflects antioxidant action of various substances, including albumin, uric acid, vitamin $\mathrm{C}$, retinol and $\alpha$-tocopherol, and depends mainly on nutritional status. ${ }^{20}$ The TAS levels in able-bodied sedentary males and able-bodied rugby players were alike but were higher in wheelchair athletes compared with sedentary individuals with tetraplegia. It cannot be excluded that these differences are caused by personal nutritional habits of the participants. The increased TAS concentration in sportsmen compared with sedentary control group has been also shown for canoeists and kayakers. ${ }^{7}$

With no data in literature concerning the effect of training on resting blood antioxidant systems in wheelchair rugby players, this study is the first to conduct an evaluation of wheelchair rugby training-induced adaptations to oxidative stress. In the present study, it was shown that adaptive response to training was similar in both able-bodied and wheelchair rugby players, and it was characterized by increased erythrocyte CAT and GPX activities in resting conditions. Nevertheless it is difficult to explain the decreased SOD activity in able-bodied rugby players, and further studies are needed to gain a better understanding of the adaptive response of antioxidant enzymatic systems to rugby training.

Our study, the first attempt to compare resting erythrocyte antioxidant enzymes activity and plasma TAS level in sedentary and rugby-trained individuals with tetraplegia with sedentary and rugbytrained able-bodied males, has some limitations. First, although the commercially available kits are commonly used in research, especially in Europe, it has to be noted that they have not been carefully validated. A second limitation is age difference between groups RA and RT, however, it should not affect our results, because the changes in erythrocyte antioxidant enzyme activates occur at older age (50-60 years).

\section{DATA ARCHIVING}

There were no data to deposit.

\section{CONFLICT OF INTEREST}

These authors declare no conflict of interest.

\section{ACKNOWLEDGEMENTS}

This study was supported by grant No DS-119 from the Ministry of Science and Higher Education in Poland. We wish to thank Elżbieta Bączkowska-Pośnik for her excellent technical assistance in the data collection.

1 Morgulec N, Kosmol A, Vanlandewijck Y, Hübner-Woźniak E. Anaerobic performance of active and sedentary male individuals with quadriplegia. APAQ 2005; 22: 253-264.

2 Gabbett T, Kinf T, Jenkins D. Applied physiology of rugby league. Sports Med 2008; 38 : 119-138.

3 Radak Z, Chung HY, Goto S. Systemic adaptation to oxidative challenge induced by regular exercise. Free Radic Biol Med 2008; 44: 153-159.

4 Brites FD, Evelson PA, Cristiansen MG, Nicol MF, Asilico MJ, Wikinski RW et al. Soccer players under regular training show oxidative stress but not improved plasma antioxidant status. Clin Sci 1999; 96: 381-385.

5 Bloomer RJ, Goldfarb AH. Anaerobic exercise and oxidative stress: a review. Can J App Physiol 2004; 29: 245-263.

6 Radak Z, Chung HY, Koltai E, Taylor AW, Goto S. Exercise, oxidative stress and hormesis. Ageing Res Rev 2008; 7: 34-42.

7 Finaud J, Lac G, Falaire E. Oxidative stress. Relationship with exercise and training. Sports Med 2006; 36: 327-358.

8 Valko M, Leibfritz D, Moncol J, Cronin MT, Mazur M, Telser J. Free radicals and antioxidants in normal physiological functions and human disease. Int J Biochem Cell Biol 2007; 39: 44-84.

9 Price M. Energy expenditure and metabolism during exercise in persons with spinal cord injury. Sports Med 2010; 40: 681-696.

10 Barfield JP, Malone LA, Arbo C, Jung AP. Exercise intensity during wheelchair rugby players. J Sports Sci 2010; 28: 389-398.

11 Panotopoulos G, Ruiz JC, Guy-Grand B, Basdevant A. Dual X-ray absorptiometry, bioelectrical impedance, and near infrared interactance in obese women. Med Sci Sports Exerc 2001; 33: 665-670.

$12 \mathrm{Ji}$ LL. Oxidative stress during exercise: implication of antioxidant nutrients. Free Radic Biol Med 1995; 18: 1079-1086. 
13 Teixeira V, Valente H, Casal S, Marquez F, Moreira P. Antioxidant status, oxidative stress and damage in elite trained kayakers and canoeists and sedentary control. Int J Sport Nutr Metabol 2009; 19: 443-456.

14 Melikoglu MA, Kaldirimci M, Katkat D, Sen I, Kaplan I, Senel K. The effect of regular long term training on antioxidant enzymatic activities. J Sports Med Phys Fitness 2008; 48: 388-390.

15 Finaud J, Scislowski V, Lac G, Durand D, Vidalin H, Robert A, Filaire E. Antioxidant status and oxidative stress in professional rugby players: evolution throughout a season. Int J Sports Med 2006; 27: 87-93.

16 Evelson P, Gambino G, Travacio M, Jaita G, Verona J, Maroncelli C et al. Higher antioxidant defences in plasma and low density lipoproteins from rugby players. Eur $J$ Clin Invest 2002; 32: 818-825.
17 Alhammari E, Shafi S, Nurmi-Lawton J, Taylor A, Lanham-New S, Ferns G. Altered antioxidant and trace elements status in adolescent female gymnasts. Int J Sport Nutr Exerc Metabol 2010; 20: 291-298.

18 Neubauer O, König D, Kern N, NIcs L, Wagner KH. No indications of persistent oxidative stress in response to an Ironman triathlon. Med Sci Sports Exerc 2008; 40: 2119-2128.

19 Goto S, Radak Z. Hormetic effects of reactive oxygen species by exercise: a view from animal studies for successful aging in human. Dose Response 2010; 8: 68-72.

20 Miller NJ, Rice-Evans C, Davies MJ, Gopinathan V, Milner A. A novel method for measuring antioxidant capacity and its application to monitoring the antioxidant status in premature neonates. Clin Sci 1993; 84: 407-412. 